\title{
Co 12029 (KARAN 13): A NEW MIDLATE SUGARCANE VARIETY RELEASED FOR COMMERCIAL CULTIVATION IN NORTH WEST ZONE OF INDIA
}

The ICAR-Sugarcane Breeding Institute (SBI), Regional Centre, Karnal (Haryana) has recently evolved a high yielding midlate maturing sugarcane variety, Co 12029 (Karan 13) for commercial cultivation in North West Zone (NWZ) of India. Co 12029 was selected from the seedling progenies of an early maturing sugarcane variety Co 0118 GC at ICAR-SBI, Regional Centre, Karnal in 2008-09. This clone was assigned 'Co' number in the year 2012, based on its superior performance for cane yield, juice quality and resistance to red rot. Co 12029 was identified for release in NWZ of India by the Varietal Identification Committee during the $32^{\text {nd }}$ Biennial Workshop of All India Coordinated Research Project (AICRP) on sugarcane held on 17 October 2018 at UAS, Bengaluru. This variety was released by the Central Varietal Release Committee during April 2019 and notified in the Gazette of India (S.O. 1498 (E) dated $1^{\text {st }}$ April 2019) for commercial cultivation in NWZ of India comprising the states of Haryana, Punjab,
Rajasthan, Central and Western Uttar Pradesh and Uttarakhand.

\section{Botanical description:}

Co 12029 is an erect growing, medium tall (stalk length $227.65 \mathrm{~cm}$ ) and medium thick cane (stalk dia $2.38 \mathrm{~cm}$ ) variety. Leaf blade curvature is mostly erect, ligule shape is crescent, inner auricle shape is mostly incipient (if present it may be deltoid). Leaf sheath colour is purplish green with medium wax. Dewlap colour may vary from pale green to yellowish green. Internode shape is mostly cylindrical (bobbin in few internodes). Internodes colour exposed to sun is grayed purple. Co 12029 exhibits shallow bud grooves, mild zigzag (internode) alignment and free from internode split. Co 12029 bears small round buds with wings, bud tip often touches growth ring. Swollen growth ring may be noticed in few canes. The rind surface is mostly smooth (ivory marks may be present on few internodes). Co 12029 does not possess spines on leaf sheath.

Table 1. Yield and quality of Co 12029 in AVT trials conducted at 26 locations in NWZ of India during 2016 to 2018 in comparison with standards (mean of $2 \mathrm{P}+1 \mathrm{R}$ )

\begin{tabular}{lcccc}
\multicolumn{1}{c}{ Characters } & \multirow{2}{*}{ Co 12029 } & \multicolumn{3}{c}{ Midlate Standards } \\
& & CoPant 97222 & CoS 767 & CoS 8436 \\
\hline $\begin{array}{l}\text { Commercial cane sugar yield (CCS } \\
\text { yield t/ha) }\end{array}$ & $\mathbf{1 2 . 0 7}$ & 10.08 & 9.47 & 8.73 \\
\% improvement over standards & & 19.74 & 27.46 & 38.26 \\
Cane yield (t/ha) & $\mathbf{9 5 . 5 8}$ & 80.97 & 78.07 & 69.16 \\
\% improvement over standards & & 18.04 & 22.43 & 38.20 \\
Sucrose \% & $\mathbf{1 8 . 1 0}$ & 17.91 & 17.58 & 18.15 \\
\% increase or decrease over standards & & 1.51 & 2.96 & $=0.28$ \\
Pol \% in cane & $\mathbf{1 4 . 1 7}$ & 13.91 & 13.39 & 13.94 \\
\% improvement over standards & & 1.87 & 5.83 & 1.65 \\
\hline
\end{tabular}




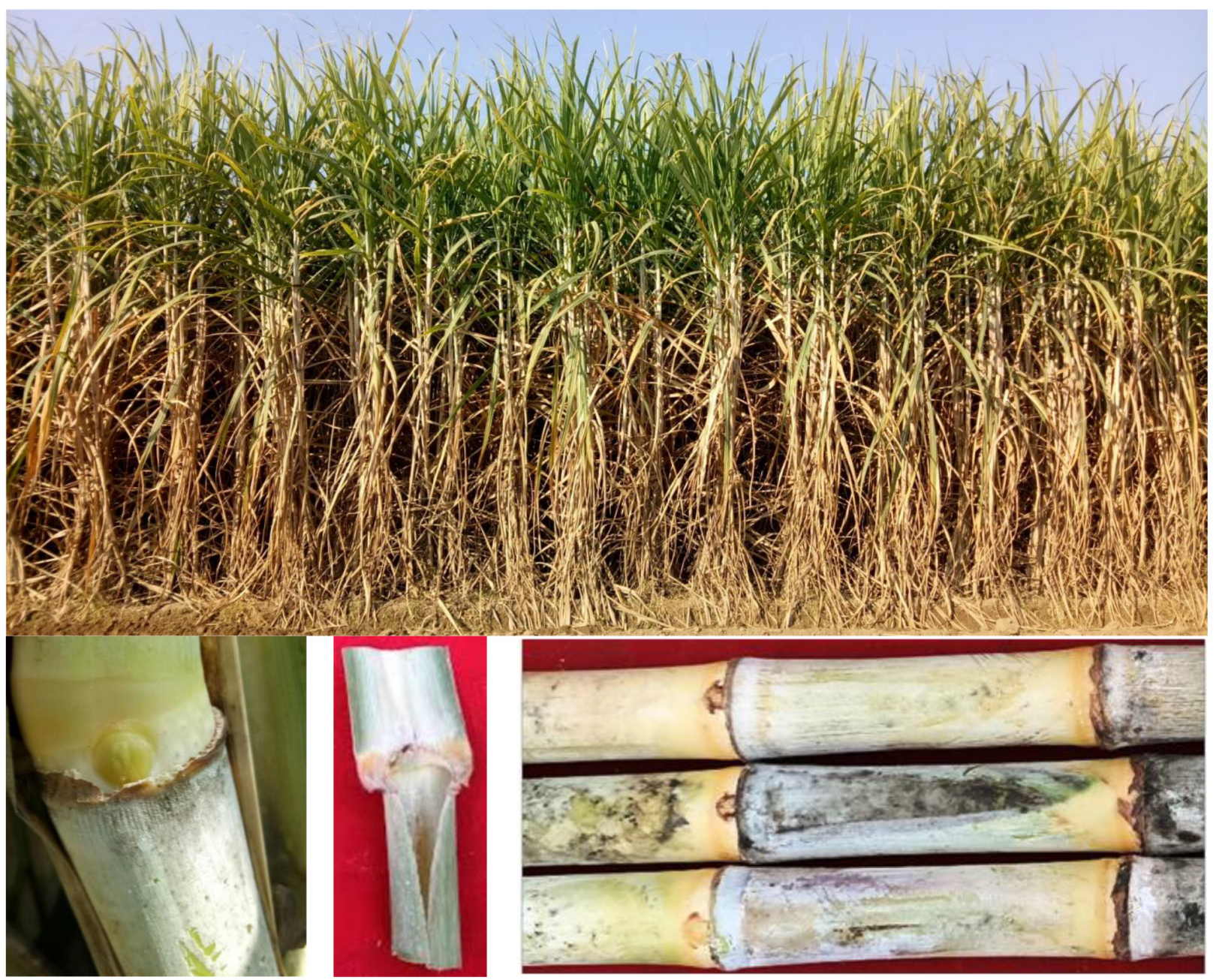

Fig 1. Morphological Description of Co 12029 (Karan 13)

Field stand, bud shape, dew-lap color, inter-node shape

\section{Performance of Co 12029}

Co 12029 was evaluated in $\operatorname{AICRP}(S)$ trials at 9 locations in North West Zone of India from 201516 to $2017-18$ (9 trials in IVT during 2015-16 and 26 trials in AVT during 2016-17 and 201718). The performance of Co 12029 in AVT trials in NWZ in comparison to the midlate standards (CoPant 97222, CoS 767 and CoS 8436) are given in Table 1.

Sugar and cane yield: Co 12029 recorded higher sugar yield and cane yield over the zonal standards.
The mean commercial cane sugar (CCS) yield of Co 12029 in NWZ was 12.07 t/ha which was 19.74 , 27.46 and 38.26 per cent improvement over the zonal standards CoPant 97222, CoS 767 and CoS 8436, respectively (Table 1). Among top 3 entries for CCS yield in the zone, Co 12029 figured in 21 out of 26 trials. Co 12029 showed superiority over the qualifying variety, $\operatorname{CoS} 12232$ for CCS yield. There was 7.70 per cent improvement in Co 12029 over CoS 12232.

The average cane yield of Co 12029 in the zone was $95.58 \mathrm{t} / \mathrm{ha}$, which is higher under subtropical 
conditions. This variety exhibited 18.04, 22.43, 38.20 per cent improvement for cane yield over the standards CoPant 97222, CoS 767 and CoS 8436, respectively. Co 12029 was in top 3 ranks for cane yield in 19 out of 26 trials in NWZ. Co 12029 showed 7.55 per cent higher cane yield over the qualifying variety $\operatorname{CoS} 12232$.

Juice quality: The average sucrose \% of Karan 13 in NWZ was 18.10 which was 1.51 and 2.96 percent improvement over the standards CoPant 97222 and $\operatorname{CoS} 767$, respectively (Table 1). Sucrose $\%$ of Co 12029 was at par with that of CoS 8436 (18.15\%). The Pol\% in cane in Co 12029 was $14.17 \%$ which was $1.87,1.65$ and 5.83 per cent improvement over the zonal standards $\mathrm{CoS}$ 8436, CoPant 97222 and $\operatorname{CoS} 767$, respectively.

Ratoon: Co 12029 is a good ratooner. It recorded an average cane yield of $89.38 \mathrm{t} / \mathrm{ha}$ in AICRP trials conducted in NWZ, which was 18.53, 20.96 and 51.06 per cent higher over the zonal standards CoS 767 (75.41 t/ha), CoPant 97222 (73.89 t/ ha) and $\operatorname{CoS} 8436$ (59.17 t/ha), respectively. The Pol \% in cane in ratoon crop of Co 12029 was $13.93 \%$, which was $4.27,5.45$ and 6.34 per cent improvement over zonal standards $\operatorname{CoS} 8436$ (13.36\%), CoPant 97222 (13.21\%) and CoS 767 (13.10\%), respectively.

Reactions against diseases and pests: Co 12029 showed mostly R (resistant) to MR (moderately resistant) reaction against $\mathrm{CF} 08$ and $\mathrm{CF} 09$ pathotypes of Colletotrichum falcatum which causes red rot disease in sugarcane. In plug method of inoculation Co 12029 showed R, MR, $\mathrm{S}$ and HS reactions to CF08, CF09 pathotypes in 5, 28, 1 and 1 out of 36 trials, respectively. In nodal cotton swab method, Co 12029 exhibited $\mathrm{R}$ or MR reaction in 34 out of 36 trials. On the basis of natural incidence, Co 12029 was rated as $\mathrm{R}$ for wilt, R or MR for yellow leaf disease (YLD) and MS for smut. Karan 13 was found 'least susceptible' variety for shoot borer, top borer and stalk borer.

Recommendation: Co 12029 is a high cane and sugar yielding midlate variety. It is a suitable variety for autumn and spring season planting, at $90 \mathrm{~cm}$ row spacing, in fertile soil, under normal environment, irrigated condition in North West Zone of India. About 30-35 quintal seed cane is required for planting one acre. It may serve as good substitute for CoPant 97222, $\mathrm{CoS} 767, \mathrm{CoS}$ 8436 which are deteriorating in the zone due to increased incidence of GSD, red rot, etc. Its profuse tillering, non-lodging, better ratoonability and stay green characteristics will certainly contribute higher cane yield and higher income to the farmers of North West Zone of India.

\section{Developers}

Bakshi Ram, R. Karuppaiyan, Mintu Ram Meena, Ravinder Kumar, Neeraj Kulshreshtha, G. Hemaprabha

\section{Colloborators}

M.L. Chhabra, B. Parameswari, S.K. Pandey 